Chinese Journal of Organic Chemistry

ARTICLE

\title{
串联式三组分反应构建取代吡唑杂环
}

李玉峰* 黄佳维顾嘉超黄浩钮长盛 马鸿飞

(南京工业大学化学与分子工程学院 南京 211816)

\begin{abstract}
摘要 建立一种串联三组分反应构建取代吡唑杂环的方法. 以肼和醛为原料在二氯乙烷中反应先生成腙 $\mathbf{3}$, 直接加入 丙炔酸甲酯(4), 在氯化亚铜催化下快速生成 Michael 加成产物 5. 该中间体以化学量的三氯化铁氧化发生环合反应, 获 得高收率的吡唑环衍生物 6. 各步反应中间体不必分离，脂肪肼、芳香肼以及芳香醛对该反应具有良好适应性.

关键词 肼; 醛; 丙炔酸甲酯; 吡唑; 三组分反应
\end{abstract}

\section{Sequential Three-Component Reactions for the Assembly of Functionalized Pyrazoles}

\author{
Li, Yufeng* \\ Huang, Jiawei \\ Gu, Jiachao \\ Huang, Hao \\ Niu, Changsheng \\ Ma, Hongfei \\ (College of Chemistry and Molecular Engineering, Nanjing Tech University, Nanjing 211816)
}

\begin{abstract}
A sequential three-component reaction for the assembly of functionalized pyrazoles is described. Aldehydes are treated with hydrazines in dichloroethane to generate hydrazones (3), which in situ take place aza-Michael reaction quickly with methyl propiolate (4) catalyzed by $\mathrm{CuCl}$ to give Michael adducts (5). Subsequent oxidation of 5 with stoichiometric $\mathrm{FeCl}_{3}$ provides substituted pyrazoles with high to excellent yields. The intermediates of the synthetic method are not necessary to isolate. Aliphatic hydrazines, aromatic hydrazines as well as aromatic aldehydes are well adaptable to the reaction.
\end{abstract}

Keywords hydrazine; aldehyde; methyl propiolate; pyrazoles; three-component reaction

吡唑环是一类十分重要的氮杂环, 在医药、农药、 材料化学合成等领域都有着广泛的应用 ${ }^{[1 \sim 9]}$. 例如, 一 些含有芳基取代吡唑结构的化合物是 HSP 90 (Heat shock protein 90)的抑制剂 ${ }^{[10]}$, 其特殊的结构使其具有 抗癌活性. 以吡唑环为母核的农药具有的内吸性高、毒 性低、高效、结构变化多样等特点. 在农药的设计与结 构拓展方面有着重要的意义. 例如, 5-酰胺基吡唑类化 合物是一种快速、高效的杀虫、杀螨剂 ${ }^{[11]}$; 氟虫腈及其 类似物是一类含有吡唑杂环结构的杀虫剂, 能有效防治 对常规杀虫剂有抗药性的刺吸式和咀嚼式口器害虫 ${ }^{[12]}$; 含吡唑的磺酰艮类化合物具有超高效的除草活性, 对环 境友好, 对农作物安全, 而且对后茬作物无影响 ${ }^{[13]}$.

迄今为止, 许多吡坐类杂环的合成方法被报 道 ${ }^{[14 \sim 19]}$, 但一锅法制备取代吡唑环的方法较少. Ma 等 ${ }^{[20]}$ 报道了腙与丁炔二酸二甲酯在 $\mathrm{Cu}(\mathrm{I})$ 催化下生成四 取代吡唑环, 然而该方法中的炔模块局限于丁炔二酸二 甲脂, 因此产物结构的多样性受到限制. Yavari 等 ${ }^{[21]}$ 在
2010 年报道了在三苯基膦催化下, 氯代腙衍生物与丙 炔酸甲酯反应制备三取代吡唑化合物的方法，但是氯代 腙衍生物不易得到. Safaei 等 ${ }^{[22]}$ 在 2012 年报道了在无溶 剂条件下, 利用离子液体催化醛、肼以及丙炔酸甲酯反 应制备吡唑环衍生物的方法, 但离子液体较为昂贵. Ji 等 ${ }^{[23]}$ 在 2013 年报道了在醋酸钯催化下, 利用等物质的 量的氧气、丙烯酸甲酯、苯胺以及苯甲腈反应制备吡唑 环衍生物的方法, 方法新颖, 但是反应过程复杂, 且催 化剂较为昂贵.

在一步反应中即可形成多个化学键, 中间体不用分 离, 是多组分反应的绿色化学特征之一. 在过去几年中,

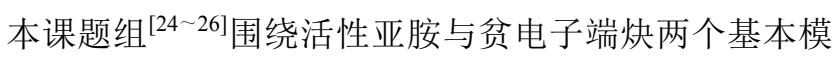
块建立了一系列新型多组分反应, 获得了多种氮杂环的 合成方法. 在这些反应中, 丙炔酸甲酯对亚胺进行加成 形成的炔丙基胺类中间体是设计串联式反应构建氮杂 环的关键中间体. 由此我们曾经设想: 丙炔酸甲酯(4)与 腙 3a 的加成反应可生成炔丙基胇衍生物, 其后, 只要条

*E-mail: yufengli@njtech.edu.cn

Received September 18, 2015; revised November 12, 2015; published online December 7, 2015. 
件得当，炔丙基肼中间体可发生氧化环合得到吡唑环 (如 Scheme 1, Supposed model). 然而, 实验结果表明, 腙 $3 \mathrm{a}$ 与丙炔酸甲酯(4)反应得到的并非是炔丙基肼, 而 是 Michael 加成产物 5a. 进一步研究发现, 中间体 5a 可 以通过氧化关环得到吡唑衍生物 $\mathbf{6 a}$ (如 Scheme 1, Actual model).

\section{1 结果与讨论}

\section{1 催化剂、溶剂、温度对生成中间体 $5 a$ 的影响}

我们首先以苯肼和苯甲醛制得的苯腙和丙炔酸甲 酯为底物, 1,2-二氯乙烷(DCE)为溶剂, 固定其投料比为 苯腙 $(3 \mathbf{a})$ : 丙炔酸甲酯 $(4)=1 ： 1$ (物质的量比), 考察了 多种催化剂包括 $\mathrm{CuI} 、 \mathrm{CuBr} 、 \mathrm{CuCl} 、 \mathrm{CuCl}_{2} 、 \mathrm{ZnCl}_{2} 、 \mathrm{FeCl}_{3}$ 、 $\mathrm{FeCl}_{2} 、 \mathrm{PdCl}_{2} 、 \mathrm{AgNO}_{3}$ 对反应的影响, 反应结果见表 1 . 当 使用 $\mathrm{CuCl}_{2} 、 \mathrm{ZnCl}_{2} 、 \mathrm{FeCl}_{2} 、 \mathrm{PdCl}_{2} 、 \mathrm{AgNO}_{3}$ 作为催化剂 时(表 1, Entries 1 5), 回流条件下反应 $5 \mathrm{~h}$, 无 $\mathbf{5 a}$ 生成. 当使用 $\mathrm{CuI} 、 \mathrm{CuBr} 、 \mathrm{CuCl}$ 和 $\mathrm{FeCl}_{3}$ 催化反应时(表 1 , Entries 6 9), 得到了中间体 $\mathbf{5 a}$, 经层析提纯并进行了结构表 征. 从表中可以看出 $\mathrm{CuCl}$ 的催化效果显著优于其它催 化剂.

在确定催化剂后, 对溶剂和温度进行了优化. 用甲 苯、 $N, N$-二甲基甲酰胺(DMF)和乙醇作溶剂时都不利于 5a 的生成(表 1, Entries 10 12). 以乙腈作溶剂时, 5a 收 率也显著降低(表 1, Entry 13). 固定 1,2-二氯乙烷为溶 剂, 降低反应温度, 对 5a 的产率不利(表 1, Entries 14 16). 因此, 以 $\mathrm{CuCl}$ 为催化剂, 1,2-二氯乙烷作溶剂, 回 流温度为腙与丙炔酸甲酯加成反应的最佳条件.

\section{2 中间体 $5 a$ 关环反应条件优化}

接着, 以 $5 \mathbf{a}$ 为底物, 对环合反应的催化(或氧化)剂 及反应条件进行了优化. 反应体系中加入 $\mathrm{CuCl}$ 、新制
表 1 反应条件对 aza-Michael 加成的影响

Table 1 Effects of experimental conditions on aza-Michael addition

\begin{tabular}{|c|c|c|c|}
\hline Entry & Catalyst $^{a}$ & Conditions & Yield $^{b} / \%$ \\
\hline 1 & $\mathrm{CuCl}_{2}$ & DCE, $80{ }^{\circ} \mathrm{C}, 5 \mathrm{~h}$ & 0 \\
\hline 2 & $\mathrm{ZnCl}_{2}$ & DCE, $80{ }^{\circ} \mathrm{C}, 5 \mathrm{~h}$ & 0 \\
\hline 3 & $\mathrm{FeCl}_{2}$ & DCE, $80{ }^{\circ} \mathrm{C}, 5 \mathrm{~h}$ & 0 \\
\hline 4 & $\mathrm{PdCl}_{2}$ & DCE, $80{ }^{\circ} \mathrm{C}, 5 \mathrm{~h}$ & 0 \\
\hline 5 & $\mathrm{AgNO}_{3}$ & DCE, $80{ }^{\circ} \mathrm{C}, 5 \mathrm{~h}$ & 0 \\
\hline 6 & $\mathrm{CuI}$ & DCE, $80{ }^{\circ} \mathrm{C}, 5 \mathrm{~h}$ & 12 \\
\hline 7 & $\mathrm{CuBr}$ & DCE, $80{ }^{\circ} \mathrm{C}, 5 \mathrm{~h}$ & 35 \\
\hline 8 & $\mathrm{CuCl}$ & DCE, $80{ }^{\circ} \mathrm{C}, 5 \mathrm{~h}$ & 88 \\
\hline 9 & $\mathrm{FeCl}_{3}$ & $\mathrm{DCE}, 80^{\circ} \mathrm{C}, 5 \mathrm{~h}$ & 23 \\
\hline 10 & $\mathrm{CuCl}$ & $\mathrm{EtOH}, 80{ }^{\circ} \mathrm{C}, 5 \mathrm{~h}$ & 42 \\
\hline 11 & $\mathrm{CuCl}$ & $\mathrm{DMF}, 100{ }^{\circ} \mathrm{C}, 5 \mathrm{~h}$ & 0 \\
\hline 12 & $\mathrm{CuCl}$ & Toluene, $100{ }^{\circ} \mathrm{C}, 5 \mathrm{~h}$ & Trace \\
\hline 13 & $\mathrm{CuCl}$ & $\mathrm{CH}_{3} \mathrm{CN}, 80{ }^{\circ} \mathrm{C}, 5 \mathrm{~h}$ & 75 \\
\hline 14 & $\mathrm{CuCl}$ & DCE, $60{ }^{\circ} \mathrm{C}, 5 \mathrm{~h}$ & 19 \\
\hline 15 & $\mathrm{CuCl}$ & DCE, $40{ }^{\circ} \mathrm{C}, 5 \mathrm{~h}$ & Trace \\
\hline 16 & $\mathrm{CuCl}$ & DCE, $25{ }^{\circ} \mathrm{C}, 5 \mathrm{~h}$ & 0 \\
\hline
\end{tabular}

\footnotetext{
${ }^{a}$ The dosage of catalyst is $20 \mathrm{~mol} \% \mathbf{3 a} .^{b}$ Isolated yield of $\mathbf{5 a}$.
}

$\mathrm{Ag}_{2} \mathrm{CO}_{3}{ }^{[27]} 、 \mathrm{ZnCl}_{2} 、 \mathrm{PdCl}_{2}$ 和 $\mathrm{CuI}$ 等金属化合物(表 2, Entries 1 11), 反应 $8 \mathrm{~h}$, 均无成环产物 $6 \mathbf{a}$ 产生. 加入 $\mathrm{FeCl}_{3}$ (20 mol\%)时(表 2, Entry 12), 反应 $8 \mathrm{~h}, \mathbf{6 a}$ 的产率 为 $22 \%$; 加入 $\mathrm{FeCl}_{3}(100 \mathrm{~mol} \%$ )时(表 2, Entry 13), 反应 $8 \mathrm{~h}, \mathbf{6 a}$ 的产率为 $58 \%$; 加入 $\mathrm{FeCl}_{3}$ (200 mol\%)时(表 2, Entry 14), 反应 $0.5 \mathrm{~h}$ 即可反应完，收率达到 $95 \%$; 考虑 到采用串联反应, 前面生成腙时即有水产生, 所以用 $\mathrm{FeCl}_{3} \cdot 6 \mathrm{H}_{2} \mathrm{O}$ 进行了对照试验(表 2, Entry 15), 可能由于 体系水量增加, 底物发生水解等副反应的原因, 收率有 所降低. 考虑到 $\mathrm{Ma}$ 等的工作, 我们用 $\mathrm{Cu}(\mathrm{I})$ 和 $\mathrm{NaOAc}$ 作为催化剂, 但是吡唑环的收率显著降低(表 2, Entries $16 \sim 18)$. 为了确定氧化剂的充分必要性, 用 $\mathrm{H}_{2} \mathrm{O}_{2}$ 和 $\mathrm{NaIO}_{4}$ 等作为氧化剂同样可以得到吡唑环, 但 $6 \mathbf{a}$ 收率较

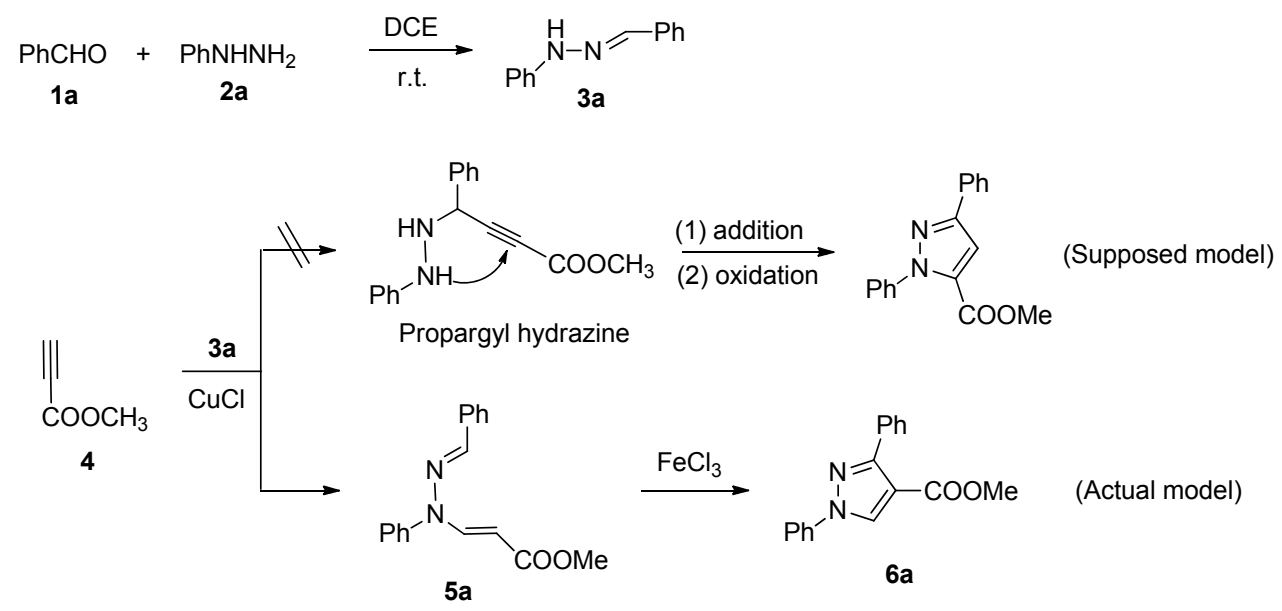

图式 $13 \mathrm{a}$ 与 4 的 Michael 加成反应与之后的环化反应

Scheme 1 Aza-Michael addition between 3a with $\mathbf{4}$ and following cyclization 
表 2 反应条件对 $5 \mathbf{a}$ 关环反应的影响

Table 2 Effects of experimental conditions on the cyclization of $5 \mathbf{a}$

\begin{tabular}{|c|c|c|}
\hline Entry & Conditions & Yield $^{a} \%$ \\
\hline 1 & $\mathrm{CuCl}(10 \mathrm{~mol} \%), \mathrm{DEE}^{b}, 35^{\circ} \mathrm{C}, 8 \mathrm{~h}$ & 0 \\
\hline 2 & $\mathrm{CuCl}(10 \mathrm{~mol} \%), \mathrm{DMF}^{c}, 80{ }^{\circ} \mathrm{C}, 8 \mathrm{~h}$ & 0 \\
\hline 3 & $\mathrm{CuCl}(10 \mathrm{~mol} \%)$, Toluene, $80{ }^{\circ} \mathrm{C}, 8 \mathrm{~h}$ & 0 \\
\hline 4 & $\mathrm{CuBr}(10 \mathrm{~mol} \%), \mathrm{DCE}^{d}, 80{ }^{\circ} \mathrm{C}, 8 \mathrm{~h}$ & 0 \\
\hline 5 & $\mathrm{CuI}(10 \mathrm{~mol} \%), \mathrm{DCE}, 80{ }^{\circ} \mathrm{C}, 8 \mathrm{~h}$ & 0 \\
\hline 6 & $\mathrm{CuCl}_{2}(10 \mathrm{~mol} \%), \mathrm{DCE}, 80{ }^{\circ} \mathrm{C}, 8 \mathrm{~h}$ & 0 \\
\hline 7 & $\mathrm{CuCl}_{2}(100 \mathrm{~mol} \%), \mathrm{DCE}, 80{ }^{\circ} \mathrm{C}, 8 \mathrm{~h}$ & 0 \\
\hline 8 & $\mathrm{FeCl}_{2}(10 \mathrm{~mol} \%), \mathrm{DCE}, 80^{\circ} \mathrm{C}, 8 \mathrm{~h}$ & 0 \\
\hline 9 & $\mathrm{ZnCl}_{2}(10 \mathrm{~mol} \%), \mathrm{DCE}, 80{ }^{\circ} \mathrm{C}, 8 \mathrm{~h}$ & 0 \\
\hline 10 & $\mathrm{Ag}_{2} \mathrm{CO}_{3}(20 \mathrm{~mol} \%), \mathrm{DCE}, 80{ }^{\circ} \mathrm{C}, 8 \mathrm{~h}$ & 0 \\
\hline 11 & $\mathrm{PdCl}_{2}(10 \mathrm{~mol} \%), \mathrm{DCE}, 80{ }^{\circ} \mathrm{C}, 8 \mathrm{~h}$ & 0 \\
\hline 12 & $\mathrm{FeCl}_{3}(20 \mathrm{~mol} \%), \mathrm{DCE}, 80{ }^{\circ} \mathrm{C}, 8 \mathrm{~h}$ & 22 \\
\hline 13 & $\mathrm{FeCl}_{3}(100 \mathrm{~mol} \%), \mathrm{DCE}, 80{ }^{\circ} \mathrm{C}, 8 \mathrm{~h}$ & 58 \\
\hline 14 & $\mathrm{FeCl}_{3}(200 \mathrm{~mol} \%), \mathrm{DCE}, 80{ }^{\circ} \mathrm{C}, 0.5 \mathrm{~h}$ & 95 \\
\hline 15 & $\mathrm{FeCl}_{3} \cdot 6 \mathrm{H}_{2} \mathrm{O}(200 \mathrm{~mol} \%)$, DCE, $80{ }^{\circ} \mathrm{C}, 0.5 \mathrm{~h}$ & 89 \\
\hline 16 & $\begin{array}{l}\mathrm{CuCl}(10 \mathrm{~mol} \%) \text {, DEE, NaOAc ( } 20 \mathrm{~mol} \%) \text {, } \\
35{ }^{\circ} \mathrm{C}, 8 \mathrm{~h}\end{array}$ & 35 \\
\hline 17 & $\mathrm{CuI}(10 \mathrm{~mol} \%)$, DEE, NaOAc, $35{ }^{\circ} \mathrm{C}, 8 \mathrm{~h}$ & 30 \\
\hline 18 & $\begin{array}{l}\mathrm{CuI}(100 \mathrm{~mol} \%) \text {, DEE, NaOAc ( } 20 \mathrm{~mol} \%) \text {, } \\
35{ }^{\circ} \mathrm{C}, 24 \mathrm{~h}\end{array}$ & 37 \\
\hline 19 & $30 \% \mathrm{H}_{2} \mathrm{O}_{2}(150 \mathrm{~mol} \%)$, DCE, $80{ }^{\circ} \mathrm{C}, 3 \mathrm{~h}$ & 75 \\
\hline 20 & $\mathrm{NaIO}_{4}(100 \mathrm{~mol} \%), \mathrm{DCE}, 80{ }^{\circ} \mathrm{C}, 3 \mathrm{~h}$ & 41 \\
\hline
\end{tabular}

${ }^{a}$ Isolated yield of 6a. ${ }^{b}$ DEE: Diethyl ether. ${ }^{c}$ DMF: $N, N$-Dimethylform-amide. ${ }^{d}$ DCE: Dichloroethane.

低(表 2, Entries 19〜20).

\section{3 串联式一锅合成法的建立及底物适应性}

为了提高产率和简化实验操作, 我们尝试建立一锅 法合成吡唑环衍生物. 以 1,2 -二氯乙烷为溶剂, 苯甲醛 $1 \mathrm{a}$ 和苯肼 $2 \mathrm{a}$ 于室温摚拌 $5 \mathrm{~min}$ 后, TLC 跟踪苯肼完全转 化完, 得到苯腙 $\mathbf{3 a}$, 不经分离提纯加入丙炔酸甲酯 $\mathbf{4}$ 和 氯化亚铜(为苯肼的 $10 \mathrm{~mol} \%$ ), 加热回流, 发生 Michael 加成反应. 当 TLC 跟踪苯腙反应完, 得到中间体 5a, 加 入化学量(2 equiv.)的氯化铁, 回流搅拌 $30 \mathrm{~min}$, TLC 跟 踪中间体 $5 \mathrm{a}$ 转化完, 分离提纯得到产物 $6 \mathrm{a}$, 产率 $87 \%$ (以苯肼计).

接着, 我们进一步考察了串联式一锅法的普适性 (表 3). 当芳香醛和芳香肼的苯环取代基为烷基、烷氧基 以及卤素时, 可以得到较高收率的吡唑环衍生物(表 3 , Entries $1 \sim 10,18$ ). 当用脂肪肼 (甲基肼)模块与各种取 代苯甲醛(包括 4-硝基苯甲醛)作用时, 经过串联反应均 顺利得到吡唑环衍生物, 收率良好(表 3, Entries 11 17).

当脂肪醛代替芳香醛与芳香肼生成腙后, 在氯化亚 铜催化下与丙炔酸甲酯在二氯乙烷中进行回流, 生成复 杂混合物, 分离困难, 且直接用三氯化铁进行关环也无
法得到相应关环产物.

如表 4 所示, 当醛类苯环上所连基团为强吸电子的 $\mathrm{NO}_{2}$ 时, 芳香肼可与其作用生成腙, 所得腙也可以与丙 炔酸甲酯在二氯乙烷回流温度下缓慢生成相应的 Michael 加成物 5, 然而该加成物无法转化为相应的吡唑 环衍生物(表 4, Entries 1 3). 当芳香肼苯环上所连基团 为强吸电子 $\mathrm{NO}_{2}$ 时, 与醛类反应所形成的腙不能形成相 应的 Michael 加成物. 其原因可能是因为强吸电子基团 存在下，使得腙中 $\mathrm{NH}$ 的亲核活性大大降低(表 4, Entries $4 \sim 5$ ).

\section{4 反应机理}

结合相关文献[28，29], 我们对反应机理进行了探 讨, 可能的机理如 Scheme 2 所示.
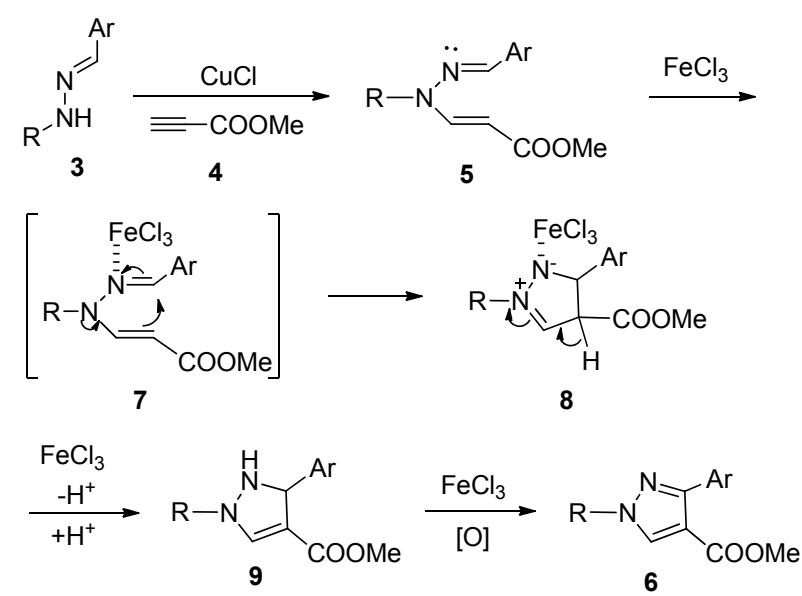

图式 2 由 3 转化到 6 的反应机理

Scheme 2 Probable mechanism of the conversion from 3 to 6

如 Scheme 2 所示, 肼与芳香酫常温下快速反应生 成腙 3 , 腙 3 与丙炔酸甲酯(4)在 1,2-二氯乙烷回流温度 下, $\mathrm{CuCl}$ 催化发生 Michael 加成反应生成中间体 5. 紧接 着, 氯化铁作为 Lewis 酸, 接受 $\mathbf{5}$ 中氮原子的配位形成 7 , 配位后的氮原子缺少电子, 引发电子转移, 发生加成 型环合反应形成中间体 $\mathbf{8}$, 中间体 $\mathbf{8}$ 发生质子迁移, 形 成中间体 9 , 迅速被氧化剂氧化得到稳定的吡唑杂环 6 . 据文献报道, 与 9 具有类似结构的化合物具有相对稳定 性 ${ }^{[22]}$, 但可能由于其氧化速度很快, TLC 方法未能检测 到其存在. 我们尝试用非氧化性的 Lewis 酸三氯化铝代 替氯化铁在惰性氛围下进行 $\mathbf{5 a}$ 的环合反应, 用 TLC 方 法可观测到有一新物质生成, 但在过滤及旋转过程中即 部分转变成 6.

\section{2 结论}

我们通过肼、酫和丙炔酸甲酯三组分串联反应建立 了一种高效的构建吡唑类杂环的方法. 该方法使用简单 易得原料, 中间体不必分离, 所合成产物具有良好的侧 
表 3 吡唑衍生物的合成 ${ }^{a}$

Table 3 Synthesis of pyrazole derivatives

\begin{tabular}{|c|c|c|c|c|c|c|c|}
\hline Entry & Structure & Product & Yield $^{b} / \%$ & Entry & Structure & Product & Yield $^{b} / \%$ \\
\hline 1 & & $6 a$ & 87 & 10 & & $6 \mathbf{j}$ & 82 \\
\hline 2 & & $6 b$ & 82 & 11 & & $6 k$ & 81 \\
\hline 3 & & $6 c$ & 81 & 12 & & 61 & 80 \\
\hline 4 & & 6d & 85 & 13 & & $6 \mathrm{~m}$ & 83 \\
\hline 5 & & $6 e$ & 88 & 14 & & 6n & 83 \\
\hline 6 & & $6 f$ & 89 & 15 & & 60 & 80 \\
\hline 7 & & $6 \mathrm{~g}$ & 85 & 16 & & $6 p$ & 81 \\
\hline 8 & & $6 h$ & 84 & 17 & & $6 q$ & 80 \\
\hline 9 & & $6 \mathbf{i}$ & 83 & 18 & & $6 r$ & 82 \\
\hline
\end{tabular}

$\overline{{ }^{a}}$ Reaction conditions: aldehyde 1 (5.0 mmol), hydrazine 2 (5.0 mmol), DCE (10.0 mL), room temperature, 5 min; methyl propiolate 4 (5.0 mmol), CuCl (0.5 mmol), $80{ }^{\circ} \mathrm{C}, 3 \mathrm{~h} ; \mathrm{FeCl}_{3}(10.0 \mathrm{mmol}), 80{ }^{\circ} \mathrm{C}, 0.5 \mathrm{~h}$, under an ambient atmosphere. ${ }^{b}$ Isolated yield of 6.

基多样性, 收率良好. 本文的研究结果也说明: 结合关 键中间体的可能性质, 用串联反应探索新的多组分反应 是一种非常有用的模式.

\section{3 实验部分}

\section{1 仪器与试剂}

${ }^{1} \mathrm{H}$ NMR 和 ${ }^{13} \mathrm{C}$ NMR 用 Bruker AV-400 进行分析, 以 TMS 为内标, $\mathrm{CDCl}_{3}$ 作溶剂; 高分辨质谱使用安捷伦
6520 Q-TOF LC/MS 仪器测定, $\mathrm{CHCl}_{3}$ 作溶剂; 熔点用 X-4 精密显微熔点测定仪测定. 所用的药品和试剂均为 市售的分析纯或化学纯，除特别说明外，未经进一步处 理.

\section{2 实验方法}

\subsection{1 中间体 5a 的制备及表征}

在装有电动摚拌的 $100 \mathrm{~mL}$ 四口烧瓶中, 加入 1,2二氯乙烷 $(10 \mathrm{~mL})$ 、苯甲醛 $(\mathbf{1 a}, 5.0 \mathrm{mmol})$ 和苯肼 $(\mathbf{2 a}, 5.0$ 
表 4 醛、肼底物中强吸电子基团对反应的影响

Table 4 The effect of electron withdrawing groups on the reactions in the substrates of aldehydes and hydrazines

\begin{tabular}{lllll}
\hline \multirow{2}{*}{ Entry } & \multicolumn{3}{c}{ Reactents 1, 2 } & \multicolumn{3}{c}{$\begin{array}{c}\text { Extent of trans- } \\
\text { formation }\end{array}$} \\
\cline { 2 - 6 } & & $\mathbf{3}$ & $\mathbf{5}$ & $\mathbf{6}$ \\
\hline 1 & $p$-Nitrobenzaldehyde, phenylhydrazine & $\sqrt{ }$ & $\sqrt{ }$ & $\times$ \\
2 & $p$-Nitrobenzaldehyde, $p$-tolylhydrazine & $\sqrt{ }$ & $\sqrt{ }$ & $\times$ \\
3 & $m$-Nitrobenzaldehyde, $p$-tolylhydrazine & $\sqrt{ }$ & $\sqrt{ }$ & $\times$ \\
4 & Benzaldehyde, (4-nitrophenyl)-hydrazine & $\sqrt{ }$ & $\times$ & $\times$ \\
& $\begin{array}{l}p \text {-Methylbenzaldehyde, } \\
\text { (4-nitrophenyl)hydrazine }\end{array}$ & $\sqrt{ }$ & $\times$ & $\times$ \\
\hline
\end{tabular}

$\mathrm{mmol}$ ), 室温搅拌 $5 \mathrm{~min}$ 后, TLC 跟踪至肼与醛已完全反 应为苯腙 $(3 \mathrm{a})$. 然后加入丙炔酸甲酯 $(\mathbf{4}, 5.0 \mathrm{mmol})$ 和氯 化亚铜 $(0.5 \mathrm{mmol})$, 加热至 $80{ }^{\circ} \mathrm{C}$, 约 $3 \mathrm{~h}$ 后, TLC 跟踪至 腙已全部消耗时反应结束, 冷却过滤, 滤液浓缩, 粗产 物经柱层析 $[V$ (石油醚) $: V($ 乙酸乙酯 $)=100: 1]$ 分离, 可得纯化产物 5a, 白色固体, 产率 88\%. m.p. 95 $97{ }^{\circ} \mathrm{C} ;{ }^{1} \mathrm{H}$ NMR $\left(400 \mathrm{MHz}, \mathrm{CDCl}_{3}\right) \delta: 8.14(\mathrm{~d}, J=13.6$ $\mathrm{Hz}, 1 \mathrm{H}), 7.63 \sim 7.53(\mathrm{~m}, 3 \mathrm{H}), 7.52 \sim 7.46(\mathrm{~m}, 3 \mathrm{H}), 7.32 \sim$ $7.29(\mathrm{~m}, 2 \mathrm{H}), 7.22 \sim 7.17(\mathrm{~m}, 3 \mathrm{H}), 4.88(\mathrm{~d}, J=13.6 \mathrm{~Hz}$, $1 \mathrm{H}), 3.69$ (s, 3H).

3.2.2 一锅法合成吡唑环衍生物 6 的典型操作

按上述中间体 5a 制备方法, 加入丙炔酸酯至腙完 全消耗后, 冷却至室温, 直接加入氯化铁 $(10.0 \mathrm{mmol})$, 回流摚拌半小时, TLC 跟踪至中间体 5a 已完全转化为产 物. 反应液过滤除去固体催化剂, 滤液浓缩, 粗产物经 柱层析 $[V$ (石油醚) $: V($ 乙酸乙酯 $)=100 ： 1]$ 分离, 可得 纯化产物 6a, 计算产率，目标产物通过 ${ }^{1} \mathrm{H} N M R 、{ }^{13} \mathrm{C}$ NMR 和 HRMS 进行结构表征.

1,3-二苯基吡唑-4-甲酸甲酯(6a): 白色固体, 产率 $87 \%$. m.p. $117 \sim 119{ }^{\circ} \mathrm{C}$ (文献值 ${ }^{[21]}: 112 \sim 114{ }^{\circ} \mathrm{C}$ ); ${ }^{1} \mathrm{H}$ NMR (400 MHz, $\left.\mathrm{CDCl}_{3}\right) \delta: 8.48(\mathrm{~s}, 1 \mathrm{H}), 7.88(\mathrm{dd}, J=6.8$, $0.6 \mathrm{~Hz}, 2 \mathrm{H}), 7.76$ (dd, $J=8.6,1.0 \mathrm{~Hz}, 2 \mathrm{H}), 7.47$ (d, $J=7.5$ $\mathrm{Hz}, 2 \mathrm{H}), 7.45 \sim 7.40$ (m, 3H), 7.33 (t, $J=7.4 \mathrm{~Hz}, 1 \mathrm{H}), 3.80$ (s, 3H); ${ }^{13} \mathrm{C}$ NMR (101 MHz, $\left.\mathrm{CDCl}_{3}\right) \delta: 163.38,154.08$, $139.24,132.32,132.05,129.60,129.35,128.76,127.97$, $127.53,119.53,113.33,51.48$. HRMS (ESI) calcd for $\mathrm{C}_{17} \mathrm{H}_{14} \mathrm{~N}_{2} \mathrm{O}_{2}[\mathrm{M}+\mathrm{H}]^{+}$279.1134, found 279.1141.

1-(4-甲基苯基)-3-苯基吡唑-4-甲酸甲酯(6b): 白色 固体, 产率 88\%. m.p. $157 \sim 159{ }^{\circ} \mathrm{C} ;{ }^{1} \mathrm{H}$ NMR $(400 \mathrm{MHz}$, $\left.\mathrm{CDCl}_{3}\right) \delta: 8.46(\mathrm{~s}, 1 \mathrm{H}), 7.87(\mathrm{dd}, J=8.0,1.5 \mathrm{~Hz}, 2 \mathrm{H}), 7.65$ (d, $J=8.5 \mathrm{~Hz}, 2 \mathrm{H}), 7.47 \sim 7.39(\mathrm{~m}, 3 \mathrm{H}), 7.28$ (d, $J=8.2$ $\mathrm{Hz}, 2 \mathrm{H}), 3.82$ (s, 3H), 2.40 (s, 3H); ${ }^{13} \mathrm{C}$ NMR (101 MHz, $\left.\mathrm{CDCl}_{3}\right) \delta: 163.45,153.87,137.49,137.00,132.22,132.17$, $130.09,129.37,128.69,127.95,119.45,113.04,51.42$, 21.01. HRMS (ESI) calcd for $\mathrm{C}_{18} \mathrm{H}_{16} \mathrm{~N}_{2} \mathrm{O}_{2}[\mathrm{M}+\mathrm{H}]^{+}$
293.1290, found 293.1296.

1-(4-氯苯基)-3-苯基吡唑-4-甲酸甲酯(6c)：白色固 体, 产率 89\%. m.p. 171 173 ${ }^{\circ} \mathrm{C} ;{ }^{1} \mathrm{H}$ NMR $(400 \mathrm{MHz}$, $\left.\mathrm{CDCl}_{3}\right) \delta: 8.48(\mathrm{~s}, 1 \mathrm{H}), 7.85(\mathrm{dd}, J=7.8,1.7 \mathrm{~Hz}, 2 \mathrm{H})$, $7.75 \sim 7.70(\mathrm{~m}, 2 \mathrm{H}), 7.47(\mathrm{~d}, J=2.2 \mathrm{~Hz}, 1 \mathrm{H}), 7.45(\mathrm{~d}, J=$ $2.0 \mathrm{~Hz}, 2 \mathrm{H}), 7.43$ (dd, $J=7.3,1.5 \mathrm{~Hz}, 2 \mathrm{H}), 3.82(\mathrm{~s}, 3 \mathrm{H})$; ${ }^{13} \mathrm{C}$ NMR (101 MHz, $\left.\mathrm{CDCl}_{3}\right) \delta: 163.19,154.29,137.75$, $133.12,132.21,131.81,129.71,129.32,128.89,127.99$, $120.62,113.70,51.52$. HRMS (ESI) calcd for $\mathrm{C}_{17} \mathrm{H}_{13} \mathrm{Cl}-$ $\mathrm{N}_{2} \mathrm{O}_{2}[\mathrm{M}+\mathrm{H}]^{+}$313.0744, found 313.0749.

1-(3,5-二甲基苯基)-3-苯基吡唑-4-甲酸甲酯(6d): 白色固体, 产率 84\%. m.p. 194 196 ${ }^{\circ} \mathrm{C} ;{ }^{1} \mathrm{H}$ NMR (400 $\left.\mathrm{MHz}, \mathrm{CDCl}_{3}\right) \delta: 8.47(\mathrm{~s}, 1 \mathrm{H}), 7.86(\mathrm{dd}, J=8.0,1.5 \mathrm{~Hz}$, 2H), $7.47 \sim 7.38$ (m, 5H), 6.99 (s, 1H), 3.81 (s, 3H), 2.39 (s, 6H); ${ }^{13} \mathrm{C}$ NMR $\left(101 \mathrm{MHz}, \mathrm{CDCl}_{3}\right) \delta: 163.42,153.93$, $139.50,139.15,132.37,132.15,129.36,129.20,128.68$, $127.94,117.37,113.02,51.41,21.37$. HRMS (ESI) calcd for $\mathrm{C}_{19} \mathrm{H}_{18} \mathrm{~N}_{2} \mathrm{O}_{2}[\mathrm{M}+\mathrm{H}]^{+}$307.1447, found 307.1454.

1-苯基-3-(4-甲基苯基)吡唑-4-甲酸甲酯(6e): 白色 固体，产率 $81 \%$. m.p. $132 \sim 134{ }^{\circ} \mathrm{C}$ (文献值 ${ }^{[21]}$ : 103 $\left.104{ }^{\circ} \mathrm{C}\right) ;{ }^{1} \mathrm{H}$ NMR $\left(400 \mathrm{MHz}, \mathrm{CDCl}_{3}\right) \delta: 8.49$ (s, 1H), 7.77 $(\mathrm{d}, J=7.8 \mathrm{~Hz}, 4 \mathrm{H}), 7.51 \sim 7.44(\mathrm{~m}, 2 \mathrm{H}), 7.37 \sim 7.32(\mathrm{~m}$, $1 \mathrm{H}), 7.27 \sim 7.23(\mathrm{~m}, 2 \mathrm{H}), 3.81(\mathrm{~s}, 3 \mathrm{H}), 2.40(\mathrm{~s}, 3 \mathrm{H}) ;{ }^{13} \mathrm{C}$ NMR $\left(101 \mathrm{MHz}, \mathrm{CDCl}_{3}\right) \delta: 163.43,154.13,139.27$, $138.63,132.25,129.58,129.23,129.15,128.70,127.44$, $119.49,113.22,51.45), 21.43$. HRMS (ESI) calcd for $\mathrm{C}_{18} \mathrm{H}_{16} \mathrm{~N}_{2} \mathrm{O}_{2}[\mathrm{M}+\mathrm{H}]^{+}$293.1290, found 293.1293.

1-苯基-3-(4-甲氧基苯基)吡唑-4-甲酸甲酯(6f)：白 色固体，产率 85\%. m.p. $146 \sim 148{ }^{\circ} \mathrm{C} ;{ }^{1} \mathrm{H}$ NMR (400 $\left.\mathrm{MHz}, \mathrm{CDCl}_{3}\right) \delta: 8.48(\mathrm{~s}, 1 \mathrm{H}), 7.91 \sim 7.80(\mathrm{~m}, 2 \mathrm{H}), 7.80 \sim$ $7.73(\mathrm{~m}, 2 \mathrm{H}), 7.47(\mathrm{t}, J=8.0 \mathrm{~Hz}, 2 \mathrm{H}), 7.34(\mathrm{t}, J=7.4 \mathrm{~Hz}$, $1 \mathrm{H}), 7.03 \sim 6.93(\mathrm{~m}, 2 \mathrm{H}), 3.85$ (s, 3H), $3.82(\mathrm{~s}, 3 \mathrm{H}) ;{ }^{13} \mathrm{C}$ NMR $\left(101 \mathrm{MHz}, \mathrm{CDCl}_{3}\right) \delta: 163.49,160.10,153.82$, $139.26,132.28,130.67,129.57,127.41,124.54,119.45$, 113.43, 112.98, 55.32, 51.44. HRMS (ESI) calcd for $\mathrm{C}_{18} \mathrm{H}_{19} \mathrm{~N}_{2} \mathrm{O}_{3}[\mathrm{M}+\mathrm{H}]^{+}$312.1474, found 312.1477.

1-苯基-3-(4-氯苯基)吡唑-4-甲酸甲酯(6g)：白色固 体, 产率 82\%. m.p. $124 \sim 126{ }^{\circ} \mathrm{C}$ (文献值 ${ }^{[21]}$ : $149{ }^{\circ} \mathrm{C}$ ); ${ }^{1} \mathrm{H}$ NMR $\left(400 \mathrm{MHz}, \mathrm{CDCl}_{3}\right) \delta: 8.49(\mathrm{~s}, 1 \mathrm{H}), 7.85(\mathrm{~d}, J=8.4$ $\mathrm{Hz}, 2 \mathrm{H}), 7.75(\mathrm{~d}, J=8.0 \mathrm{~Hz}, 2 \mathrm{H}), 7.48(\mathrm{t}, J=7.8 \mathrm{~Hz}, 2 \mathrm{H})$, $7.44 \sim 7.32(\mathrm{~m}, 3 \mathrm{H}), 3.82(\mathrm{~s}, 3 \mathrm{H}) ;{ }^{13} \mathrm{C}$ NMR $(101 \mathrm{MHz}$, $\left.\mathrm{CDCl}_{3}\right) \delta: 163.25,152.88,139.12,134.79,132.46,130.70$, 130.52, 129.64, 128.19, 127.68, 119.52, 113.28, 51.55. HRMS (ESI) calcd for $\mathrm{C}_{17} \mathrm{H}_{13} \mathrm{ClN}_{2} \mathrm{O}_{2}[\mathrm{M}+\mathrm{H}]^{+} 313.0744$, found 313.0737 . 
1-(4-甲基苯基)-3-(2-甲基苯基)吡唑-4-甲酸甲酯 (6h): 白色固体, 产率 85\%. m.p. $180 \sim 182{ }^{\circ} \mathrm{C} ;{ }^{1} \mathrm{H}$ NMR $\left(400 \mathrm{MHz}, \mathrm{CDCl}_{3}\right) \delta: 8.47(\mathrm{~s}, 1 \mathrm{H}), 7.64(\mathrm{~d}, J=8.5 \mathrm{~Hz}$, $2 \mathrm{H}), 7.36(\mathrm{~d}, J=7.5 \mathrm{~Hz}, 1 \mathrm{H}), 7.34 \sim 7.27(\mathrm{~m}, 3 \mathrm{H}), 7.26(\mathrm{~s}$, $1 \mathrm{H}), 7.26 \sim 7.22(\mathrm{~m}, 1 \mathrm{H}), 3.72(\mathrm{~s}, 3 \mathrm{H}), 2.40(\mathrm{~s}, 3 \mathrm{H}), 2.28$ (s, 3H); ${ }^{13} \mathrm{C}$ NMR $\left(101 \mathrm{MHz}, \mathrm{CDCl}_{3}\right) \delta: 163.21,154.08$, 137.41, 137.31, 137.06, 132.27, 130.98, 130.21, 130.07, $129.86,128.60,125.21,119.39,114.43,51.31,20.99$, 20.03. HRMS (ESI) calcd for $\mathrm{C}_{19} \mathrm{H}_{18} \mathrm{~N}_{2} \mathrm{O}_{2}[\mathrm{M}+\mathrm{H}]^{+}$ 307.1447, found 307.1453 .

1-(4-氯苯基)-3-(4-甲氧基苯基)吡唑-4-甲酸甲酯 (6i): 白色固体, 产率 $83 \%$. m.p. $168 \sim 170{ }^{\circ} \mathrm{C} ;{ }^{1} \mathrm{H}$ NMR $\left(400 \mathrm{MHz}, \mathrm{CDCl}_{3}\right) \delta: 8.45(\mathrm{~s}, 1 \mathrm{H}), 7.83(\mathrm{~d}, J=8.8 \mathrm{~Hz}$, 2H), $7.72(\mathrm{~d}, J=8.8 \mathrm{~Hz}, 2 \mathrm{H}), 7.45(\mathrm{~d}, J=8.8 \mathrm{~Hz}, 2 \mathrm{H}), 6.98$ (d, $J=8.8 \mathrm{~Hz}, 2 \mathrm{H}), 3.86(\mathrm{~s}, 3 \mathrm{H}), 3.82(\mathrm{~s}, 3 \mathrm{H}) ;{ }^{13} \mathrm{C} \mathrm{NMR}$ $\left(101 \mathrm{MHz}, \mathrm{CDCl}_{3}\right) \delta: 163.30,160.20,154.03,137.78$, $132.98,132.18,130.64,129.68,124.28,121.24,120.54$, 113.46, 55.32, 53.44, 51.49. HRMS (ESI) calcd for $\mathrm{C}_{18} \mathrm{H}_{15} \mathrm{ClN}_{2} \mathrm{O}[\mathrm{M}+\mathrm{H}]^{+}$311.0951, found 311.0956.

1-(3,5-二甲基苯基)-3-(2-氯苯基)吡唑-4-甲酸甲酯 (6j): 黄色固体, 产率 $82 \%$. m.p. $201 \sim 203{ }^{\circ} \mathrm{C} ;{ }^{1} \mathrm{H}$ NMR $\left(400 \mathrm{MHz}, \mathrm{CDCl}_{3}\right) \delta: 8.48(\mathrm{~s}, 1 \mathrm{H}), 7.48$ (ddd, $J=5.3,3.0$, $1.7 \mathrm{~Hz}, 2 \mathrm{H}), 7.38(\mathrm{~s}, 2 \mathrm{H}), 7.37 \sim 7.31(\mathrm{~m}, 2 \mathrm{H}), 6.99(\mathrm{~s}$, $1 \mathrm{H}), 3.74(\mathrm{~s}, 3 \mathrm{H}), 2.38(\mathrm{~s}, 6 \mathrm{H}) ;{ }^{13} \mathrm{C}$ NMR $(101 \mathrm{MHz}$, $\left.\mathrm{CDCl}_{3}\right) \delta: 163.04,151.73,139.54,139.12,134.30,132.11$, $131.48,131.06,129.90,129.34,129.28,126.42,117.43$, 115.04, 51.43, 21.34. HRMS (ESI) calcd for $\mathrm{C}_{18} \mathrm{H}_{15} \mathrm{ClN}_{2} \mathrm{O}_{3}$ $[\mathrm{M}+\mathrm{H}]^{+}$343.0849, found 343.0855.

1-甲基-3-苯基吡夾-4-甲酸甲酯(6k): 黄色固体, 产 率 81\%. m.p. 104 106 ${ }^{\circ} \mathrm{C}$ (文献值 ${ }^{[21]}$ : $35{ }^{\circ} \mathrm{C}$ ); ${ }^{1} \mathrm{H}$ NMR $\left(400 \mathrm{MHz}, \mathrm{CDCl}_{3}\right) \delta: 7.95(\mathrm{~s}, 1 \mathrm{H}), 7.80 \sim 7.72(\mathrm{~m}, 2 \mathrm{H})$, $7.43 \sim 7.35$ (m, 3H), 3.95 (s, 3H), 3.77 (s, 3H); ${ }^{13} \mathrm{C}$ NMR $\left(101 \mathrm{MHz}, \mathrm{CDCl}_{3}\right) \delta: 163.44,153.28,135.74,132.32$, $129.16,128.83,128.44,127.89,125.77,111.39,51.22$, 39.31. HRMS (ESI) calcd for $\mathrm{C}_{12} \mathrm{H}_{12} \mathrm{~N}_{2} \mathrm{O}_{2}[\mathrm{M}+\mathrm{H}]^{+}$ 217.0977, found 217.0981.

1-甲基-3-(2-氯苯基)吡唑-4-甲酸甲酯(61): 黄色固 体, 产率 80\%. m.p.143 145 ${ }^{\circ} \mathrm{C} ;{ }^{1} \mathrm{H}$ NMR $(400 \mathrm{MHz}$, $\left.\mathrm{CDCl}_{3}\right) \delta: 7.95(\mathrm{~s}, 1 \mathrm{H}), 7.45(\mathrm{dd}, J=7.6,1.6 \mathrm{~Hz}, 1 \mathrm{H})$, $7.42 \sim 7.38(\mathrm{~m}, 1 \mathrm{H}), 7.36 \sim 7.28(\mathrm{~m}, 2 \mathrm{H}), 3.97(\mathrm{~s}, 3 \mathrm{H})$, 3.69 (s, 3H); ${ }^{13} \mathrm{C}$ NMR $\left(101 \mathrm{MHz}, \mathrm{CDCl}_{3}\right) \delta: 163.07$, $150.86,134.43,134.16,132.28,131.38,129.70,129.28$, $126.33,113.58,51.24,39.40$. HRMS (ESI) calcd for $\mathrm{C}_{12} \mathrm{H}_{11} \mathrm{ClN}_{2} \mathrm{O}_{2}[\mathrm{M}+\mathrm{H}]^{+}$251.0587, found 251.0592.

1-甲基-3-(2-甲基苯基)吡唑-4-甲酸甲酯(6m): 黄色
固体, 产率 83\%. m.p. $150 \sim 152{ }^{\circ} \mathrm{C} ;{ }^{1} \mathrm{H}$ NMR (400 MHz, $\left.\mathrm{CDCl}_{3}\right) \delta: 7.95(\mathrm{~s}, 1 \mathrm{H}), 7.31 \sim 7.27(\mathrm{~m}, 1 \mathrm{H}), 7.26(\mathrm{~s}, 1 \mathrm{H})$, 7.21 (dd, $J=15.1,7.6 \mathrm{~Hz}, 2 \mathrm{H}), 3.96$ (s, 3H), 3.67 (s, 3H), $2.21(\mathrm{~s}, 3 \mathrm{H}) ;{ }^{13} \mathrm{C}$ NMR $\left(101 \mathrm{MHz}, \mathrm{CDCl}_{3}\right) \delta: 163.26$, $153.38,137.21,134.54,132.51,130.02,129.73,128.40$, $125.15,112.88,51.14,39.30,19.87$. HRMS (ESI) calcd for $\mathrm{C}_{13} \mathrm{H}_{14} \mathrm{~N}_{2} \mathrm{O}_{2}[\mathrm{M}+\mathrm{H}]^{+}$231.1134, found 231.1129.

1-甲基-3-(4-甲氧基苯基)吡唑-4-甲酸甲酯(6n): 白 色固体, 产率 $80 \%$. m.p. $117 \sim 119{ }^{\circ} \mathrm{C} ;{ }^{1} \mathrm{H}$ NMR (400 $\left.\mathrm{MHz}, \mathrm{CDCl}_{3}\right) \delta: 7.92(\mathrm{~s}, 1 \mathrm{H}), 7.76 \sim 7.70(\mathrm{~m}, 2 \mathrm{H}), 6.97 \sim$ $6.91(\mathrm{~m}, 2 \mathrm{H}), 3.93(\mathrm{~s}, 3 \mathrm{H}), 3.84(\mathrm{~s}, 3 \mathrm{H}), 3.77(\mathrm{~s}, 3 \mathrm{H}) ;{ }^{13} \mathrm{C}$ NMR $\left(101 \mathrm{MHz}, \mathrm{CDCl}_{3}\right) \delta: 163.55,159.83,153.06$, $140.55,135.72,130.44,124.82,113.35,111.00,55.27$, $51.19,39.27$. HRMS (ESI) calcd for $\mathrm{C}_{13} \mathrm{H}_{14} \mathrm{~N}_{2} \mathrm{O}_{3}[\mathrm{M}+\mathrm{H}]^{+}$ 247.1083, found 247.1090.

1-甲基-3-(4-氯苯基)吡唑-4-甲酸甲酯(60): 黄色固 体, 产率 83\%. m.p. 131 $133{ }^{\circ} \mathrm{C} ;{ }^{1} \mathrm{H}$ NMR $(400 \mathrm{MHz}$, $\left.\mathrm{CDCl}_{3}\right) \delta: 7.94(\mathrm{~s}, 1 \mathrm{H}), 7.74(\mathrm{~d}, J=8.4 \mathrm{~Hz}, 2 \mathrm{H}), 7.38(\mathrm{~d}$, $J=8.4 \mathrm{~Hz}, 2 \mathrm{H}), 3.95$ (s, 3H), 3.77 (s, 3H); ${ }^{13} \mathrm{C}$ NMR $(101$ $\left.\mathrm{MHz}, \mathrm{CDCl}_{3}\right) \delta: 163.31,152.08,135.87,134.45,130.80$, 130.50, 128.10, 111.44, 51.28, 39.36. HRMS (ESI) calcd for $\mathrm{C}_{12} \mathrm{H}_{11} \mathrm{ClN}_{2} \mathrm{O}_{2}[\mathrm{M}+\mathrm{H}]^{+}$251.0587, found 251.0593.

1-甲基-3-(4-甲基苯基)吡唑-4-甲酸甲酯(6p): 黄色 固体, 产率 80\%. m.p. $125 \sim 127{ }^{\circ} \mathrm{C} ;{ }^{1} \mathrm{H}$ NMR $(400 \mathrm{MHz}$, $\left.\mathrm{CDCl}_{3}\right) \delta: 7.93(\mathrm{~s}, 1 \mathrm{H}), 7.65(\mathrm{~d}, J=8.1 \mathrm{~Hz}, 2 \mathrm{H}), 7.21(\mathrm{~d}$, $J=7.9 \mathrm{~Hz}, 2 \mathrm{H}), 3.93$ (s, 3H), 3.76 (s, 3H), 2.38 (s, 3H); ${ }^{13} \mathrm{C}$ NMR $\left(101 \mathrm{MHz}, \mathrm{CDCl}_{3}\right) \delta: 163.50,153.34,138.24$, $135.69,129.40,129.02,128.62,111.25,51.19,39.28$, 21.35. HRMS (ESI) calcd for $\mathrm{C}_{13} \mathrm{H}_{14} \mathrm{~N}_{2} \mathrm{O}_{2}[\mathrm{M}+\mathrm{H}]^{+}$ 231.1134, found 231.1142.

1-甲基-3-(4-硝基苯基)吡唑-4-甲酸甲酯(6q): 黄色 固体, 产率 81\%. m.p. $141 \sim 143{ }^{\circ} \mathrm{C} ;{ }^{1} \mathrm{H}$ NMR $(400 \mathrm{MHz}$, $\left.\mathrm{CDCl}_{3}\right) \delta: 8.27(\mathrm{~d}, J=1.9 \mathrm{~Hz}, 1 \mathrm{H}), 8.25(\mathrm{~d}, J=2.0 \mathrm{~Hz}$, $1 \mathrm{H}), 8.03(\mathrm{~d}, J=2.0 \mathrm{~Hz}, 1 \mathrm{H}), 8.01(\mathrm{~d}, J=1.9 \mathrm{~Hz}, 1 \mathrm{H}), 7.99$ (s, 1H), 3.99 (s, 3H), 3.80 (s, 3H); ${ }^{13} \mathrm{C}$ NMR (101 MHz, $\left.\mathrm{CDCl}_{3}\right) \delta: 163.05,150.77,138.77,136.23,131.22,130.01$, $123.13,112.07,51.50,39.55$. HRMS (ESI) calcd for $\mathrm{C}_{12} \mathrm{H}_{11} \mathrm{~N}_{3} \mathrm{O}_{4}[\mathrm{M}+\mathrm{H}]^{+}$262.0828, found 262.0833 .

1-苯基-3-(3-甲基苯基)吡唑-4-甲酸甲酯(6r): 白色 固体, 产率 82\%. m.p. 119 121 ${ }^{\circ} \mathrm{C} ;{ }^{1} \mathrm{H}$ NMR $(400 \mathrm{MHz}$, $\left.\mathrm{CDCl}_{3}\right) \delta: 8.41(\mathrm{~s}, 1 \mathrm{H}), 7.73 \sim 7.64(\mathrm{~m}, 2 \mathrm{H}), 7.58(\mathrm{~d}, J=$ $8.8 \mathrm{~Hz}, 2 \mathrm{H}), 7.40$ (dd, $J=10.7,5.1 \mathrm{~Hz}, 2 \mathrm{H}), 7.26(\mathrm{td}, J=$ 7.5, $2.9 \mathrm{~Hz}, 2 \mathrm{H}), 7.18 \sim 7.12(\mathrm{~m}, 1 \mathrm{H}), 3.73(\mathrm{~s}, 3 \mathrm{H}), 2.34(\mathrm{~s}$, $3 \mathrm{H}) ;{ }^{13} \mathrm{C}$ NMR $\left(101 \mathrm{MHz}, \mathrm{CDCl}_{3}\right) \delta: 162.32,153.17$, $138.19,136.49,131.25,130.87,128.79,128.53,128.49$, 
$126.77,126.44,125.56,118.49,112.29,50.40,20.45$.

HRMS (ESI) calcd for $\mathrm{C}_{18} \mathrm{H}_{16} \mathrm{~N}_{2} \mathrm{O}_{2}[\mathrm{M}+\mathrm{H}]^{+}$293.1290, found 293.1298

辅助材料(Supporting Information) 中间体 $5 \mathbf{a}$ 以及产 物 6a $\sim \mathbf{6} \mathbf{r}$ 的 ${ }^{1} \mathrm{H}$ NMR 和 ${ }^{13} \mathrm{C}$ NMR 谱图. 这些材料可以 免费从本刊网站(http://sioc-journal.cn/)上下载.

\section{References}

[1] Abdel-Gawad, M. S.; Abdel-Aziem, A. M.; Ghorab, M. Phosphorus, Sulfur Silicon Relat. Elem. 2003, 178, 1793.

[2] Eriksson, I. B.; Quinlan, J. D. Drugs 2006, 66, 1410.

[3] Farghaly, A. M.; Soliman, G. S. F.; El Semary, A. M. M.; Rostom, F. A. S. Pharmazie 2001, 56, 23.

[4] Seki, K.; Isegawa, J.; Fukuda, M.; Oki, M. Chem. Pharm. Bull. 1984, 32, 1566.

[5] Cottineau, B.; Toto, P.; Marot, C.; Pipaud, A.; Chenault, J. Bioorg. Med. Chem. Lett. 2002, 12, 2102.

[6] Cardia, C. M.; Corda, L.; Fadda, A. M.; Maccioni, A. M.; Maccioni, E.; Plumitallo, A. Farmaco 1998, 53, 696.

[7] Mullican, D. M.; Wilson, W. M.; Cannor, T. D.; Kostlan, R. C.; Dyer, D. R. J. Med. Chem. 1993, 36, 1088.

[8] Rahman, M. A.; Siddiqui, A. A. Int. J. Pharm. Sci. Drug Res. 2010, 2,165 .

[9] Turechek, W. W.; Peres, N. A.; Werner, N. A. Plant Dis. 2006, 90 , 862.

[10] James, D. M.; William, D. B. WO 2003055860, 2003 [Chem. Abstr. 2003, 139, 101122].

[11] Okada, I.; Shiga, Y.; Kikutake, K. JP 2002205985, 2002 [Chem. Abstr. 2002, 137, 93748].

[12] Kyomura, N.; Fukuchi, T.; Okui, S. WO 9845274, 1998 [Chem. Abstr. 1998, 129, 302635].

[13] Yamamoto, S.; Satou, T.; Igai, T.; Oguchi, T.; Nawamaki, T. JP 60208977, 1985 [Chem. Abstr. 1985, 104, 148871].
[14] Ma, C. W.; Li, Y. M.; Wen, P.; Yan, R. L.; Ren, Z. Y.; Huang, G. S. Synlett 2011, 1321

[15] Peruncheralathan, S.; Khan, T. A.; Ila, H.; Junjappa, H. J. Org. Chem. 2005, 70, 10030.

[16] Manecke, G.; Schenck, H. U. Tetrahedron Lett. 1969, 8, 617.

[17] Wang, Z. S.; Ye, H. T.; Xiao, D.; Gao, J. S.; Yu, Y. H.; Hou, G. F.; Yan, P. F. Chin. J. Org. Chem. 2014, 34, 2057 (in Chinese). (王子时，叶汉蹈，肖丹，马东升，高金胜，于颖慧，侯广峰，问 鹏飞, 有机化学, 2014, 34, 2057.)

[18] Chen, B.; Yu, C. J.; Zhang, G. Z. Chin. J. Org. Chem. 2015, 35, 625 (in Chinese). (陈斌, 于丛军, 张国柱, 有机化学, 2015, 35, 625.)

[19] Zheng, C. Z.; Xun, X. D.; Wang, Y. Z.; Zhao, D. J.; Zhang, J. Z. Chin. J. Org. Chem. 2015, 35, 1137 (in Chinese). (郑纯智, 徐小丹, 王雅珍, 赵德建, 张继振. 有机化学, 2015 , $35,1137$.

[20] Ma, C.; Li, Y; Wen, P.; Yan, R.; Ren, Z; Huang, G. Synlett 2011, 1321.

[21] Yavri, I.; Khalili, G.; Mirzaei, A. Helv. Chim. Acta 2010, 93, 277.

[22] Safaei, S.; Mohammadpoor-Baltork, I.; Khosropour, A. R.; Moghadam, M.; Tangestaninejad, S.; Mirkhani, V. Adv. Synth. Catal. 2012, 354, 3095.

[23] Ji, X. C.; Huang, H. W.; Wu, W. Q.; Li, X. W.; Jiang, H. F. J. Org. Chem. 2013, 78, 11155.

[24] Li, Y. F.; Shi, J.; Wu, Z.; Wang, X. L.; Wu, X.; Gu, J.; Bu, H.; Ma, H. Tetrahedron 2014, 70, 2472.

[25] Li, Y. F.; Wu, Z.; Shi, J.; Pan, Y.; Bu, H. Z.; Ma, H.; Gu, J.; Huang, H.; Wang, Y.; Wu, L. Tetrahedron 2014, 70, 8971.

[26] Li, Y. F.; Wu, Z.; Shi, J.; Bu, H.; Gu, J. C.; Pan, Y. Tetrahedron 2014, 70, 3134.

[27] Zhang, L. Z.; Li, L. Q.; Ren, X. J.; Xie, Z. X. Acta Chim. Sinica 2008, 66, 589 (in Chinese). (张鲁中, 李立奇, 任小娟, 谢志翔, 化学学报, 2008, 66, 589.)

[28] Li, R. S.; Wang, S. R.; Lu, W. J. Org. Lett. 2007, 11, 2219.

[29] Yang, L.; Lei, C. H.; Wang, D. X.; Huang, Z. T.; Wang, M. X. Org. Lett. 2010, 17, 3918 . 